\title{
THE DEFORMATON OF THE GEAR HOB'S GENERATING SURFACES DUE TO RE-SHARPENING
}

\author{
Márton MÁTÉ ${ }^{1}$, Dénes HOLLANDA ${ }^{2}$ \\ Sapientia Hungarian University of Transylvania, Faculty of Technical and Human Sciences, \\ Department of Mechanical Engineering, Târgu-Mureș, Romania \\ ${ }^{1}$ mmate@ms.sapientia.ro \\ 2 hollanda@ms.sapientia.ro
}

\begin{abstract}
The side relief faces of the monolithic involute gear hob are machined through relieving. The resulting surfaces are bevel helical surfaces in which the side cutting edges result from the intersection of these with the helical rake face. Theoretically, the gear hob is derived from an involute worm. Resharpening decreases the diameter of the hob, thus the edges became closer to the axis, and as a consequence they will be situated on a smaller worm than the original. The present paper analyses the deviation of the re-sharpened gear hob's carrying worm from the theoretically perfect involute worm whose characteristic dimensions were adjusted considering the re-sharpened gear hob characteristic diameters. It was proven that the evolution of the errors is significantly different from that described in the literature. Thus, increasing the new gear hob diameters in comparison with the calculated dimensions is unnecessary, because it cannot reduce the error to half with this procedure. The mathematical model was built up accepting that the edges result from the intersection of an involute worm with a helical rake face and the side relief faces result from the rototranslation of the edges on a bevel helix leading curve dressed by the relieving parameter.
\end{abstract}

Keywords: gear-hob, involute worm, relieving, re-sharpening, deviation.

\section{The Model of the Gear Hob's Generating Worm}

According to the majority of opinions given in the literature [1-8], the gear hob's generating worm is considered in the present paper to be an involute worm. Mathematically, the helical surfaces of the involute worm can be generated using two different procedures $[1,3,4,5,6,8]$ : rototranslating generating lines, or rototranslating involutes over a cylindrical helix leader curve. The present model will make use of generating lines. In order to obtain the greatest possible simplification of the right sides of the equations, generating lines motion will start from a particular position that differs from the position of the real cutting edges in the manufacturing process $[1, \underline{3}, \underline{4}, \underline{5]}$. Figure 1. shows the basic circle's involute curves and the corresponding generatrix lines. If axis $z$ is oriented from left to right, generatrix $A_{1} A_{2}$ producing involute curve $C A_{1}$ will mesh the right worm surface. Similarly, generatrix $B_{1} B_{2}$ describing the involute $D B_{1}$ will mesh the left worm surface. The definition of left and 
right side is made for the tooth. The inclination angle $\lambda_{b}$ of the generatrix is equal to the inclination angle of the basic helix line. The pressure angle $\alpha_{o t}$ can be computed from the inclination angle $\lambda_{0}$ of the pitch helix line, the normal module $m_{n}$ and the number of teeth of the worm $i$, using the equations of the involute geometry [9]. In the present paper the generating worm has only one tooth.

The $x y z$ frame is attached to the worm. Axis $y$ is the symmetry axis of the radial section. It is easy to remark that generatrix lines can be rotated from the basic position (Figure 1.) in any arbitrary position, respecting the condition of tangency to the basic helix.

The computing and the formulas can be essentially simplified if - exploiting the property mentioned before - planes $\left(A A_{1} A_{2}\right)$ respectively $\left(B B_{1} B_{2}\right)$ containing the generating lines and tangents to the basic cylinder are roto-translated till they become parallel and both perpendicular to axis $y$. Generating line $A_{1} A_{2}$ turns in $A_{1}^{*} A_{2}^{*}$ and $B_{1} B_{2}$ in $B_{1}^{*} B_{2}^{*}$. The parameters of the roto-translation are the axial pitch $p_{a x}=\frac{\pi m_{n}}{\cos \lambda_{0}}$ and the rotation angle $\frac{\pi}{2}-\alpha_{0 t}$.

The parametric equations of the generating lines can be written using the geometrical relations shown in Figure 2:

$$
\left\{\begin{array}{l}
x(u)=R_{b} \\
y(u)=-j u \\
z(u)=j\left(h_{b}-u \operatorname{tg} l_{b}\right) \quad, j \in\{-1 ; 1\} \\
h_{b}=\frac{m_{n}}{2 \cos \lambda_{0}}\left(\frac{\pi}{2}+\operatorname{inv} \alpha_{0 t}\right)
\end{array}\right.
$$

Here the parameter $j$ is assigned to -1 for the left side and to +1 for the right side of the worm tooth.

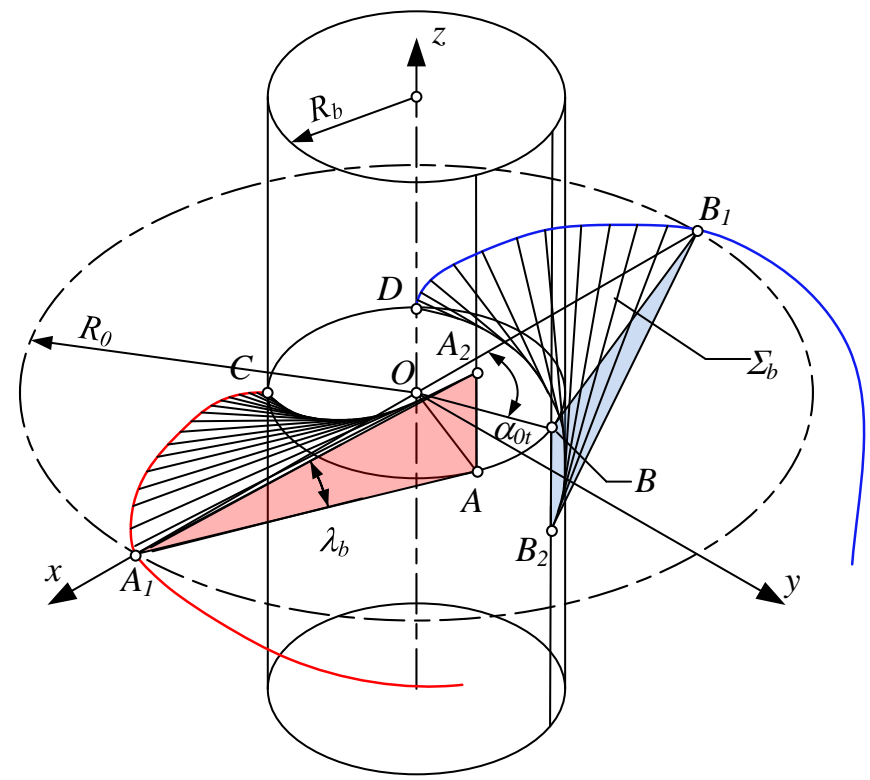

Figure 1. The radial section of the involute worm and the worm surface generating lines 


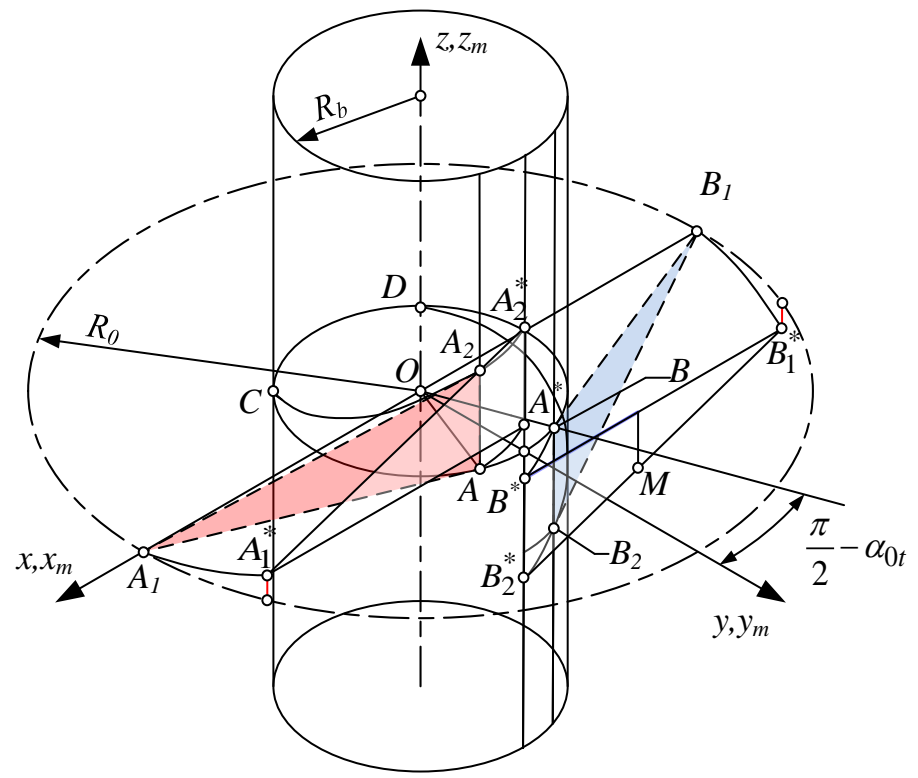

Figure 2. The generating lines of the involute worm surfaces rotated in the starting position.

The surfaces of the worm tooth are obtained if generatrix lines are attached first to a mobile frame $x_{m} y_{m} z_{m}$, initially in covering with $x y z$, followed by a roto-translation of parameter $p_{a x}$ about axis $z$. A detailed presentation is given in [8]. It is useful to keep the rotation parameter $v$ on positive values, thus the roto-translation matrix takes the following form:

$\mathbf{M}(v ; j)=\left(\begin{array}{cccc}\cos j v & -\sin j v & 0 & 0 \\ \sin j v & \cos j v & 0 & 0 \\ 0 & 0 & 1 & \frac{p_{a x}}{2 \pi} v \\ 0 & 0 & 0 & 1\end{array}\right)$

The equations of the generating worm surfaces are obtained using expressions (1) and (2) within the matrix equation of the transformation $[3,4]$ :

$$
\left\{\begin{array}{l}
x(u, v)=R_{b} \cos v+u \sin v \\
y(u, v)=j\left(R_{b} \sin v-u \cos v\right) \\
z(u, v)=j\left(h_{b}-u \operatorname{tg} \lambda_{b}+\frac{p_{a z}}{2 \pi} v\right)
\end{array}\right.
$$

Parameter $h_{b}$ in the expression of the $z$ coordinate signifies the distance of points $A_{2}^{*}$ respectively $B_{2}^{*}$ from the plane $(x y)$ (Figure 2). Its value results from geometric dependencies of the basic helix line and the involute:

$$
h_{b}=\frac{m_{n}}{2 \cos \lambda_{0}}\left(\frac{\pi}{2}+\operatorname{inv} \alpha_{0 t}\right)
$$

Equations (3) present an advantageous form because parameters $u$ and $v$ are separated. This particularity confers a significant advantage when computing the intersection of helical surfaces with the rake face.

\section{The side relief faces of the invo- lute gear hob.}

\subsection{The relief face generating edges}

The relief face generating edges are the intersections of the theoretical (designed) involute worm and the rake face. This latter is also a helical surface whose pitch helix line is perpendicular to the pitch helix line 
of the involute worm. As a consequence, the sense of its helix is opposite to that of the worm, but the tangent line to its pitch helix forms the angle $\lambda_{0}$ with the axis $z$. The rake face generating line intersects axis $z$ staying perpendicular to it. Let's suppose that the start position of the generating line forms angle $-\varepsilon$ with axis $x$. This ensures the possibility of moving the rake face in any desired position relative to the involute worm. The basic position is given by the particular value $\varepsilon=0$ of the position parameter. The general equation of the rake face can be written on the basis of Figure 3:

$$
\left\{\begin{array}{l}
x(t, w ; \varepsilon)=t \cos (w-\varepsilon) \\
y(t, w ; \varepsilon)=t \sin (w-\varepsilon) \\
z(t, w ; \varepsilon)=-\frac{p_{C}}{2 \pi} w
\end{array}\right.
$$

The helix pitch $p_{C}$ of the rake face is computed from the condition of perpendicularity of the pitch helices [8].

In the following, the implicit form of the equation of rake face will be used:

$$
\operatorname{arctg} \frac{y}{x}+\frac{2 \pi}{p_{C}} z+\varepsilon=0
$$

As defined before, the generating curves of the side relief faces are obtained by intersecting the involute worm's surfaces with the rake face. Introducing the parametric functions (3) in equation (6) and priming $v$ depending on $u$ the edge defining function becomes as follows:

$$
\begin{array}{r}
v(u)=\frac{p_{C}}{p_{a x}+p_{C}}\left(\operatorname{arctg} \frac{u}{R_{b}}-j \varepsilon\right)+ \\
+\frac{2 \pi}{p_{a x}+p_{C}}\left(u \operatorname{tg} \lambda_{b}-h_{b}\right)
\end{array}
$$

Finally, the parametric equations of the generating edges are given by expressions (3) if parameter $v$ is replaced by function (7).

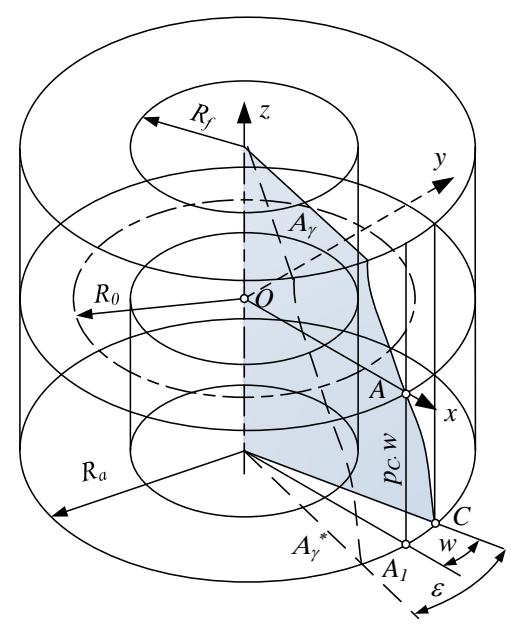

Figure 3. The rake face of the gear hob

\subsection{The equations of the side relief faces}

The side relief faces are generated by passing the generating edges over a conical helix leading line which is defined by the relieving depth $k$ and the axial pitch $p_{a x}$. The detailed model of the side relief face meshing is described in $[3,4,8]$. Using the transformation given in [8], the parametric equations of the side relief face result as follows:

$$
\left\{\begin{aligned}
x(u, \varphi ; j)= & R_{b} \cos E(u, \varphi)+u \sin E(u, \varphi)- \\
& -a \varphi \cos \varphi \\
y(u, \varphi ; j)= & j\left(R_{b} \sin E(u, \varphi)-u \cos E(u, \varphi)\right)+ \\
& +a \varphi \sin \varphi \\
z(u, \varphi ; j)= & j\left(h_{b}-u \operatorname{tg} \lambda_{b}+\frac{p_{a x}}{2 \pi} E(u, \varphi)\right) \\
E(u, \varphi)= & v(u)-j \varphi
\end{aligned}\right.
$$

\section{The meshing worm of the re- sharpened gear hob}

If the theoretical generating edges (3) with (7) are roto-translated about axis $z$ following a leader helix with axial pitch $p_{a x}$ these will re-mesh the theoretical worm surfaces from which they originate. Geometrically 
the re-sharpening can be defined as the rotation of the rake face about the axis $z$ with an angle $\varepsilon$ whose value increases with the number of re-sharpenings. Due to the conical helix effect, the new edges move closer to the axis with the distance $\delta_{\varepsilon}=\frac{k z_{m}}{2 \pi} \varepsilon$. As a consequence, they will fit a worm whose characteristic diameters decrease with $2 \delta_{\varepsilon}$. The decreasing of the diameter under constant axial pitch leads to the increasing of the pitch helix angle.

In order to compute the re-sharpened edges, the side relief face parametric functions (8) will be inserted in the rake face implicit equation (6). After elementary trigonometric transformations an implicit equation is obtained in unknowns $u, \varphi$ that meets the following form:

$$
\begin{aligned}
& \varphi+j \xi(u, \varphi)- \\
& -j \frac{2 \pi\left(h_{b}-u \operatorname{tg} \lambda_{b}\right)+p_{a x}(v(u)-j \varphi)}{p_{C}}-\varepsilon=0
\end{aligned}
$$

Equation (9) is built up by use of the following functions:

$$
\begin{aligned}
& E_{1}(u)=v(u)-\operatorname{arctg} \frac{u}{R_{b}} \\
& Q(u)=\frac{k z_{m}}{R_{b}} \varphi \cos \left(\operatorname{arctg} \frac{u}{R_{b}}\right)-\cos E_{1}(u) \\
& \xi(u, \varphi)=\operatorname{arctg} \frac{\sin E_{1}(u)}{Q(u)}
\end{aligned}
$$

It is to be observed that equation (9) is transcendent regarding both variables and as a consequence it can be only numerically solved.

The solving procedure consists in priming the value of angle $\varphi$ for a set of $N$ discrete values of parameter $u$. First of all let's remark that in using the first two parametric expressions of the involute worm (3) the distance of an arbitrary surface point to the axis $z$ of the helix can be primed as

$$
\rho=\sqrt{R_{b}^{2}+u^{2}}
$$

Only the subset of surface points situated between the addendum and the dedendum cylinder are considered. Thus, using equation (11) the limits of $u$ became:

$$
\sqrt{R_{f}^{2}-R_{b}^{2}} \leq u \leq \sqrt{R_{a}^{2}-R_{b}^{2}}
$$

Now let's define an equidistant division over the compact set of radii $\left\lfloor R_{f}, R_{a}\right\rfloor$ :

$$
\begin{aligned}
& \rho_{l}=R_{f}+l \Delta_{\rho}, \Delta_{\rho}=\left(R_{a}-R_{f}\right) /(N-1), \\
& l=0,1, \ldots, N-1
\end{aligned}
$$

Using formula (11) the equidistant radius division (13) generates a $u$ division. Solving equation (9) for each $u_{l}, l=\overline{0 . . N-1} N$ ordered pairs $\left(u_{l}, \varphi_{l}\right), l=\overline{0 . . N-1}$ are obtained. Introducing these in equations (8) of the side relief faces a set of $3 N$ coordinates result. Pairing the resulted $x, y, z$ values with the corresponding $u_{l}$ values the edges can be written as cubic spline fitting functions depending only on parameter $u$ :

$$
\left\{\begin{array}{l}
x(u)=S_{x}(u) \\
y(u)=S_{y}(u) \\
z(u)=S_{z}(u)
\end{array}\right.
$$

The meshing worm of the new edges becomes the generating surface for the machined gear. The equations of the meshing worm result from the following matrix equation:

$$
\underline{\mathbf{r}}(u, w)=\left(\begin{array}{cccc}
\cos w & -\sin w & 0 & 0 \\
\sin w & \cos w & 0 & 0 \\
0 & 0 & 1 & \frac{p_{a x}}{2 \pi} w \\
0 & 0 & 0 & 1
\end{array}\right)\left(\begin{array}{c}
S_{x}(u) \\
S_{y}(u) \\
S_{z}(u) \\
1
\end{array}\right)
$$




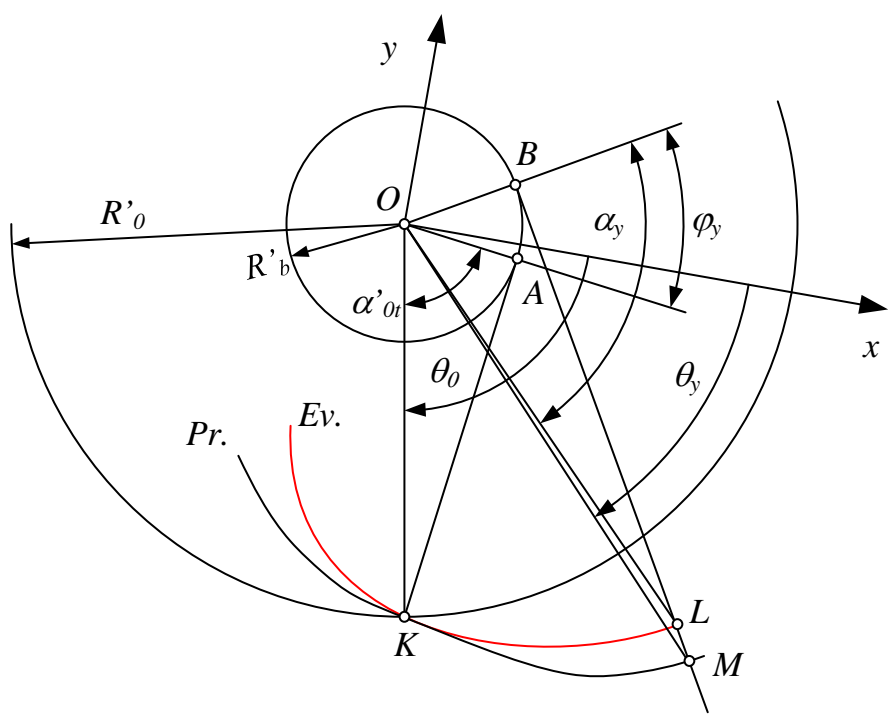

Figure 4.The definition of the deviation of the generating surface

\section{The re-sharpened gear hob fit- ting ideal involute worm}

As a consequence of the re-sharpening the pitch radius decreases to

$$
R_{0}^{\prime}=R_{0}-\delta_{e}
$$

The normal module must be kept constant (theoretically it varies due to re-sharpening [4]) thus the inclination angle of the pitch helix turns in

$$
\lambda_{0}^{\prime}=\arcsin \frac{\pi m_{n}}{2 \pi R_{0}^{\prime}}
$$

Using the new pitch radius and pitch helix angle, the basic circle radius and the involute pitch pressure angle can be computed:

$$
\begin{aligned}
& \alpha_{0 t}^{\prime}=\operatorname{arctg} \frac{\operatorname{tg} \alpha_{0 n}}{\sin \lambda_{0}^{\prime}} \\
& R_{b}^{\prime}=R_{0}^{\prime} \cos \alpha_{0 t}^{\prime}
\end{aligned}
$$

\section{The computing of the deviation}

Let's define the deviation as the distance between the radial sections of the meshing worm and the ideal involute worm of the resharpened gear hob. The geometrical formulation of the definition is shown in Figure 4. It is evident that the ideal involute worm radial section is an involute that it intersects the radial section curve of the meshing worm on the pitch circle. The distance is measured in normal direction to the involute, thus the measuring direction is a tangent to the basic circle.

The radial section of the meshing worm results from expanding matrix equation (15) followed by zeroing coordinate $z$ :

$$
\begin{aligned}
& z(u, w)=0 \Leftrightarrow S_{z}(u)+\frac{p_{a x}}{2 \pi} w=0 \\
& w(u)=-\frac{2 \pi}{p_{a x}} S_{z}(u)
\end{aligned}
$$


Replacing $w$ in the parametric functions of the coordinates $x, y$ with the function $w(u)$ the meshing worm's radial section becomes

$$
\left\{\begin{array}{l}
x(u)=S_{x}(u) \cos w(u)-S_{y}(u) \sin w(u) \\
y(u)=S_{x}(u) \sin w(u)+S_{y}(u) \cos w(u)
\end{array}\right.
$$

The value of parameter $u$ for the pitch radius results from the equation

$$
\left(R_{0}^{\prime}\right)^{2}-S_{x}^{2}(u)-S_{y}^{2}(u)=0
$$

The root of (22) will be implemented in expressions (21). The obtained $x$ and $y$ values are the coordinates of point $K$, defining a position vector having the polar angle

$$
\theta_{0}=\operatorname{arctg} \frac{y\left(u_{0}\right)}{z\left(u_{0}\right)}
$$

It should be mentioned that the present computing of angle $\theta_{0}$ is made considering the values of the arc tangent function on a symmetric interval comprising double period $[-\pi, \pi] \neg\left\{\frac{-\pi}{2} ; \frac{\pi}{2}\right\}$. If point $K$ is situated in the III or the IV quadrant, the value of the polar angle is negative, otherwise it becomes positive.

Let's consider an arbitrary point $M$ on the meshing worm's radial section and a tangent line to the basic circle, denoted $M B$. This tangent line intersects the ideal involute passing through $K$ at point $L$. The polar angle of position vector $\mathrm{OM}$ is computed similarly to (23):

$$
\theta_{y}=\operatorname{arctg} \frac{y\left(u_{M}\right)}{z\left(u_{M}\right)}
$$

The difference in lengths of tangent segments $K A$ and $L B$ - as it results from the mathematical definition of the circle's involute - is equal to the basic circle $\operatorname{arc} A B=R_{b}^{\prime} \varphi_{y}$. The expression of angle $\varphi_{y}$ can be deduced using synthetic geomet- ric properties resulting from figure 4 . It can be written that:

$$
\varphi_{y}=\arccos \frac{R_{b}^{\prime}}{O M}+\theta_{y}-\left(\theta_{0}+\alpha_{0 t}^{\prime}\right)
$$

The angle $\alpha_{y}$ is primed using the orthogonal triangle $B O M$. Finally, the expression of the deviation can be computed involving expressions $(23, . ., 25)$ in the following formula:

$$
\Delta=R_{b}^{\prime}\left(\operatorname{tg} \alpha_{y}-\operatorname{tg} \alpha_{0 t}^{\prime}-\varphi_{y}\right)
$$

The sign of the deviation shows the relative position of the meshing and the ideal involute worm. If the deviation is positive then the involute curve falls in the interior of the meshing worm's section, thus the tool eliminates more material from the tooth gape than necessary and as a consequence it produces a small and acceptable undercut of the tooth base and also a crowning on the addendum segment. If the deviation is negative, then the tool meshes a thinner tooth gap while the involute surface remains covered by the allowance rest.

\section{Numerical simulation}

The mathematical model presented in the previous chapters was tested on gear hobs with one thread, $\alpha_{0 n}=20^{\circ}$ normal involute pressure angle, and $m_{n}=5 \mathrm{~mm}$ normal module. Gear hobs were studied with pitch helix inclination angle set at $\lambda_{01}=2^{\circ}$, and $0 \lambda_{02}=3^{\circ}$. In the first case the teeth number matches $z_{m 1}=12$, while in the second case $z_{m 2}=10$. The value of the relieving parameter was computed considering the top relief angle value set on $\alpha_{V}=8^{\circ}$. While designing a gear hob, the literature recommends the increasing of its characteristic diameters corresponding to the half of the re-sharpening reserve. This reserve is considered equal to the half of the angular 
pitch. As a result the increasing of the characteristic radii must match the Archimedean spiral polar radius variation for a polar angle of $\frac{1}{2} \frac{\pi}{z_{m}}$. It can be written that

$$
\frac{1}{2} \frac{\pi}{z_{m}} \frac{k z_{m}}{2 \pi}=\frac{k}{4}
$$

It is to be pointed out that the real edges of the new gear hob are obtained from equations (9) by setting the $\varepsilon$ angle value at $-\frac{\pi}{2 z_{m}}$.

The computation of the deviation was realized for 4 states of wear. For more clarity the wear state was primed in percent. The states considered in this simulation correspond to $0,25,75$, and $100 \%$-of wear, The corresponding $\varepsilon$ values are:

$$
\varepsilon \in\left\{-\frac{\pi}{2 z_{m}} ;-\frac{\pi}{4 z_{m}} ; \frac{\pi}{4 z_{m}} ; \frac{\pi}{2 z_{m}}\right\}
$$

Here $\varepsilon=0$ is omitted until it corresponds to a $50 \%$ wear state, where characteristic diameters of the gear hob are equal to those of the theoretical generating worm and as a conclusion the deviation is zero by definition.

The repartition of the deviations are presented in Figures 5-12.

Analyzing simultaneously the deviations presented in the Figures 5-8 it can be concluded that their position and values depend on the side of the edge and the degree of wear.

It is interesting that the deviation curves in case of a new gear hob are identical for the left and the right side edges.

This results from Figure 5. where the graphic shown below indicates the differences of deviations recorded on the left and right edges. The magnitude here indicates the numerical approximation error.

Figures 6. and 7. show deviations corresponding to wear statuses situated on the opposite sides of the ideal status, and the
$25 \%$ and the $75 \%$ wear. It can be observed that the sense of curvatures changes. It should also be noted that the deviation on the right side is significantly greater than on the link side, but also that due to their magnitude, this is negligible. On the right edge the deviation is negative for radii less than the pitch radius, at $25 \%$ of wear, and positive otherwise. If the wear status reaches $75 \%$ then the sense of the repartition is inverted. Between the limits of $75 \%$ and $100 \%$ wear the repartition of the deviation keep their characteristic, but the values increase to twice.

Figures 9-12 show the repartition of the deviations in the case of a gear hob having a $3^{\circ}$ pitch helix inclination. It can be observed that all characteristics and evolution of the deviation functions are identical with those deduced for the $2^{\circ}$ inclined pitch helix.

Here the deviation of the new hob reaches $10 \mu \mathrm{m}$ that is inadmissible. In contrast to this, the full wear status corresponds to a maximal deviation of only 3 microns which can be accepted.
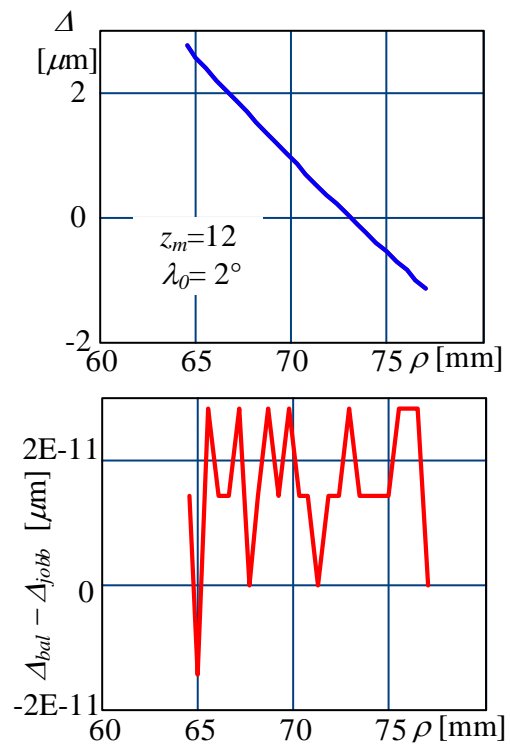

Figure 5. Deviation in the case of new gear hob 


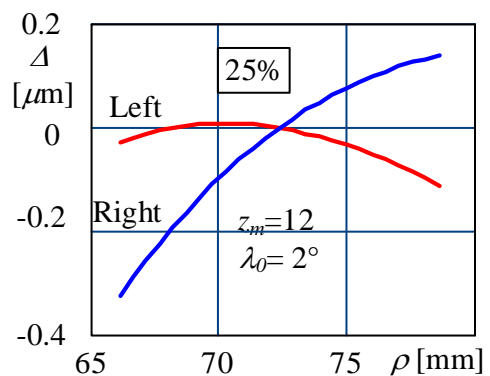

Figure 6. Deviation in the case of $25 \%$ wear

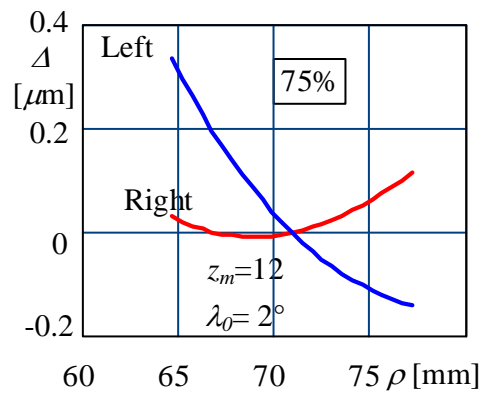

Figure 7. Deviation in the case of $75 \%$ wear

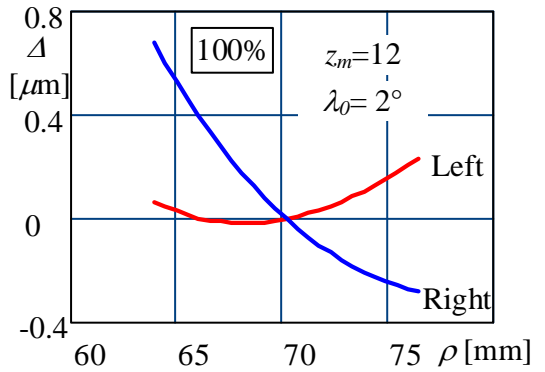

Figure 8. Deviation in the case of $100 \%$ wear

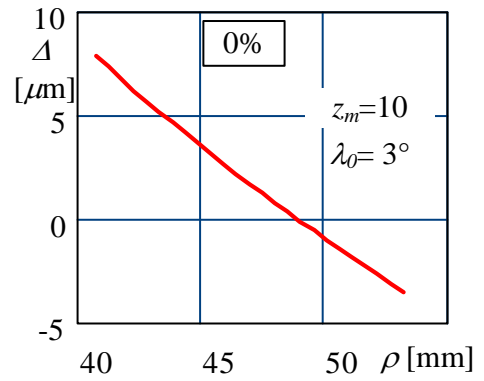

Figure 9. Deviation by a new gear hob

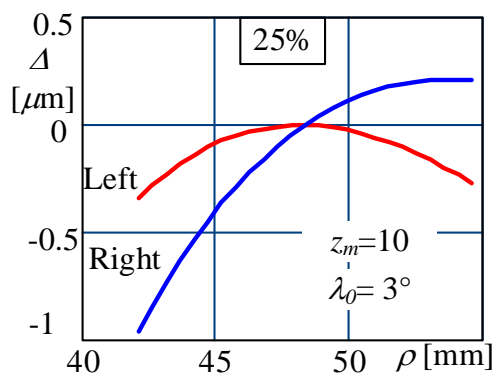

Figure 10. Deviation in case of $25 \%$ wear

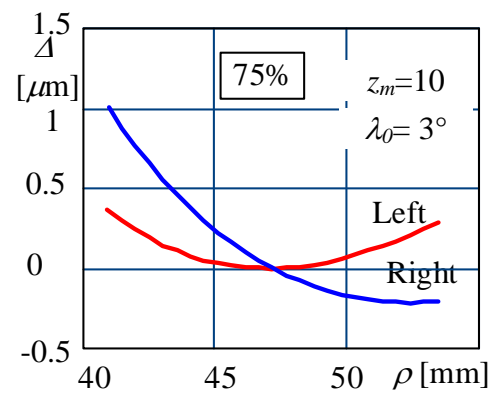

Figure 11. Deviation in the case of $75 \%$ wear

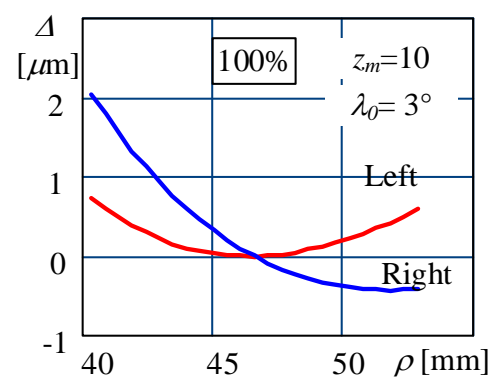

Figure 12. Deviation in the case of $100 \%$ wears

\section{Conclusions}

The numerical investigation of the gear hob's mathematical model makes it possible to formulate some conclusions regarding the construction and design of monolithic gear hobs.

First it is necessary to emphasize that despite the fact that the ideal status is situated on the middle of the re-sharpening reserve e.g. between $0 \%$ and $100 \%$ of wear, the 
deviations are significantly higher before the ideal status than after. This conclusion is supported through the comparative investigation of figures $6,7,8$, and figures 10,11 and 12 . The deviation of the $25 \%$ worn and re-sharpened $2^{\circ}$ pitch helix inclined gear hob is nearly 6 times lower in comparison with its new status. In the case of the $3^{\circ}$ pitch helix gear hob this scale increases to almost 20, which is significant!

In the wear interval of $25 \%-75 \%$ the deviation values remain under one micron. It can be concluded that they have only theoretical significance.

Even in the case of total wear status the re-sharpened gear hobs show acceptable deviation values in comparison with the ideal involute gear suited to the diminished diameters.

Considering the particularities of the deviation evolution with wear status, it can be concluded that the classical design of the gear hob - where the ideal elements are set on the middle of the wear reserve - is not the optimal solution. The conception regarding the dimension of the new gear hob must be renewed. The re-sharpening reserve must be distributed about the ideal dimensions in such way that the deviations should not exceed the admissible value. The model can be used for the computing of the limits of variation regarding the pitch diameter.
When the difference between the theoretical and the real grinding wheel meshed relief face is significant, the variation of the deviation functions can be slightly different.

\section{References}

[1] Radzevich S. P.: Dudley's Handbook of Practical Gear Design. CRC-Press, London, 2016, 368-379.

[2] Litvin F. L.: A fogaskerékkapcsolás elmélete. Müszaki Könyvkiadó, Budapest, 1972, 187190.

[3] Hollanda D.: Bazele așchierii şi generării suprafețelor. Petru Maior University, TârguMureș, 1994.

[4] Máté M.: Hengeres fogaskerekek gyártószerszámai, Erdélyi Múzeum-Egyesület, ClujNapoca, 2016, 187-206.

[5] Dudás I.: The Theory and Practice of Gear Worm Drives, Penton Press, London, 2000.

[6] Radzevich P. S.: Gear Cutting Tools. Fundamentals of design and computation, CRCPress, London, 2010.

[7] Gyenge Cs.: Lefejtömarók oldalhátszögének pontos meghatározása és optimálása, Gép, 48/10. (1996) 38-40.

[8] Máté M.: A hengeres fogaskerék-lefejtő csigamaró müködö élgeometriájának vizsgálata. In: A XVII. Müszaki tudományos ülésszak előadásai, MTK6. (2016) 137-147. http://eda.eme.ro/handle/10598/30078

[9] Szeniczei L.: Általános fogazás. Nehézipari Müszaki Könyvkiadó, Budapest, 1957. 